\title{
nArgBP2 as a hub molecule in the etiology of various neuropsychiatric disorders
}

\author{
Sang-Eun Lee E Sunghoe Chang* \\ Department of Physiology and Biomedical Sciences, Seoul National University College of Medicine, Seoul 01030, Korea
}

\begin{abstract}
Recent studies have strongly implicated postsynaptic scaffolding proteins such as SAPAP3 or Shank3 in the pathogenesis of various mood disorders, including autism spectrum disorder, bipolar disorder (BD), and obsessive-compulsive disorders. Neural Abelson-related gene-binding protein 2 (nArgBP2) was originally identified as a protein that interacts with SAPAP3 and Shank3. Recent study shows that the genetic deletion of nArgBP2 in mice leads to manic/bipolar-like behavior resembling symptoms of BD. However, the function of nArgBP2 at synapse, or its connection with the synaptic dysfunctions, is completely unknown. This study provides compelling evidence that $\mathrm{nArgBP2}$ regulates the spine morphogenesis through the activation of Rac1/WAVE/PAK/cofilin pathway, and that its ablation causes a robust and selective inhibition of excitatory synapse formation, by controlling actin dynamics. Our results revealed the underlying mechanism for the synaptic dysfunction caused by nArgBP2 downregulation that associates with analogous human BD. Moreover, since nArgBP2 interacts with key proteins involved in various neuropsychiatric disorders, our finding implies that nArgBP2 could function as a hub linking various etiological factors of different mood disorders. [BMB Reports 2016; 49(9): 457-458]
\end{abstract}

nArgBP2, a neural specific splice variant of the ubiquitous ArgBP2, belongs to a family of adaptor proteins involved in the regulation of cell adhesion, actin cytoskeleton organization,

*Corresponding author. E-mail: sunghoe@snu.ac.kr

http://dx.doi.org/10.5483/BMBRep.2016.49.9.138

Received 9 August 2016

Keywords: Actin, Bipolar disorder, Dendritic spines, Excitatory synapse, nArgBP2

Abbreviations: Arp2/3, Actin-related protein; BD, Bipolar disorder; nArgBP2, neural Abelson-related gene binding protein 2; SAPAP3, synapse-associated protein 90/postsynaptic density protein 95 -associated protein 3

Perspective to: $\mathrm{nArgBP} 2$ regulates excitatory synapse formation by controlling dendritic spine morphology. Lee SE et al. (2016) Proc. Natl. Acad. Sci. USA.;113(24):6749-54. doi:10.1073/pnas.1600944113 and signaling downstream of growth factor receptors. Members of this protein family are characterized by a sorbin homology (SoHo) domain in the $\mathrm{NH} 2$-terminal region, and three $\mathrm{SH} 3$ domains in the $\mathrm{COOH}$-terminal region. We previously reported that the $\mathrm{NH}_{2}$-terminal region of nArgBP2 interacts with spectrin, while the $\mathrm{COOH}$-terminal $\mathrm{SH} 3$ domains bind to dynamin, synaptojanin, and WAVEs as well as WAVE regulatory proteins that directly or indirectly participate in the regulation of the actin cytoskeleton. Our previous study also found that the downregulation of $\mathrm{ArgBP} 2 / \mathrm{nArgBP} 2$ expression in astrocytes increases peripheral actin ruffles, whereas nArgBP2 overexpression causes a coalescence of the actin cytoskeleton, suggesting nArgBP2 controls actin cytoskeleton dynamics.

Bipolar disorder (BD), also known as manic-depressive illness, is one of the most common, severe and devastating neuropsychiatric disorders. Despite the high prevalence and severity of $\mathrm{BD}$, little is known about its neurobiological basis. nArgBP2 mRNA is highly expressed in the isocortex, hippocampal formation, cortical subpalate, striatum, thalamus and hypothalamus, in addition to the cerebellum granular layer; majority of these are regions of the brain associated with BD. $A$ recent study showed that the genetic deletion of nArgBP2 in mice leads to manic/bipolar-like behavior including increased activity, compulsive/repetitive risk-taking and hedonistic behaviors, resembling many symptoms of BD.

nArgBP2 binds to the synapse-associated protein 90/postsynaptic density protein 95-associated protein 3 (SAPAP3) that has been implicated in the pathogenesis of obsessive-compulsive behaviors. SAPAP also binds to Shank3, a postsynaptic scaffolding protein, whose overexpression causes manic-like behaviors while its loss was linked to autism spectrum disorders. Despite the high possibility that nArgBP2 could be involved in the etiology of various mood disorders, nothing is known about the role of nArgBP2 at synapses, or its connection with the synaptic dysfunctions associated to these neurological disorders.

Immunocytochemistry was initially done to study the expression pattern of nArgBP2 in neurons. In primary cultured hippocampal neurons, nArgBP2 were primarily detected in dendrites that colocalized with PSD95 (excitatory postsynaptic protein). nArgBP2 clusters were also juxtaposed with vGLUT1 (excitatory presynaptic protein) in spiny neurons. In contrast, nArgBP2 hardly overlapped with gephyrin (inhibitory postsynaptic protein) in spiny neurons, and was almost absent in 
gephyrin-positive aspiny neurons. Thus, we conclude that nArgBP2 primarily localizes to the excitatory synapses in spiny pyramidal neurons.

To study the physiological role of nArgBP2 in neurons, we used small-hairpin RNA mediated knock-down (KD) of endogenous $\mathrm{nArgBP} 2$ in neurons. We found that along with a lower density of total dendritic protrusions in $K D$, dendritic spine morphology was strikingly affected. nArgBP2 KD resulted in a dramatic decrease of mushroom-shaped spines and a concomitant increase of long, thin, filopodia-like protrusions.

Dendritic spines are actin-rich architectures that receive presynaptic inputs in most excitatory synapses of CNS. We colabeled neurons with antibodies against vGLUT1 and PSD95, or VGAT and gephyrin, to distinguish between excitatory and inhibitory synapses, respectively. Surprisingly, the number of excitatory synaptic contacts on dendritic spines in spiny neurons was dramatically decreased in KD, although the number of vGAT-gephyrin-positive inhibitory synaptic contacts on the dendritic shaft remained unchanged. Neither excitatory nor inhibitory contacts in aspiny neurons were altered, indicating that nArgBP2 KD selectively impairs excitatory spine-synapse formation with the decrease in mushroom-shaped spines. Furthermore, the mean frequency of miniature Excitatory Postsynaptic Potential (mEPSC) was significantly reduced, while nArgBP2 KD had no effect on miniature Inhibitory Postsynaptic potential (mIPSC) in both frequency and amplitude, thus supporting its selective role in spine-bearing excitatory synapses.

Next, we studied the molecular mechanisms linking reduced nArgBP2 to aberrant spine morphology. We found that nArgBP2 binds to WAVE and to WAVE regulatory complex, which is activated by Rac1 that also promotes cofilin phosphorylation via PAK-LIMK. We observed an increased WAVE/PAK/cofilin phosphorylation, indicating that $\mathrm{nArgBP} 2 \mathrm{KD}$ causes activation of WAVE and PAK, and a subsequent inactivation of cofilin. Furthermore, we showed that inhibition of PAK by PAKinhibitory domain, or the activation of cofilin by constitutively active cofilin mutant S3A, combined with sequestration of WAVE, compensates the spine defects by nArgBP2 KD. Thus, these results demonstrate a causal relation between elevated WAVE/PAK/cofilin phosphorylation and the aberrant spine phenotype of nArgBP2 KD neurons.

To examine the actin dynamics within spines, we performed fluorescence recovery after photobleaching (FRAP) experiments. In nArgBP2 KD neurons, the proportion of dynamic actin was significantly increased, and the fraction of stable actin was concomitantly reduced. This alteration of actin dynamics correlates with the wider shape factor fluctuation which suggests irregular shapes and highly dynamic spine motility. In addition, the inhibition of Rac1 by NSC23766 successfully rescued the effect of $\mathrm{nArgBP} 2 \mathrm{KD}$ on actin composition. Together, our results showed that nArgBP2 regulates spine morphogenesis and subsequent spine-synapse formation at the glutamatergic synapse by controlling actin dynamics (Fig. 1).

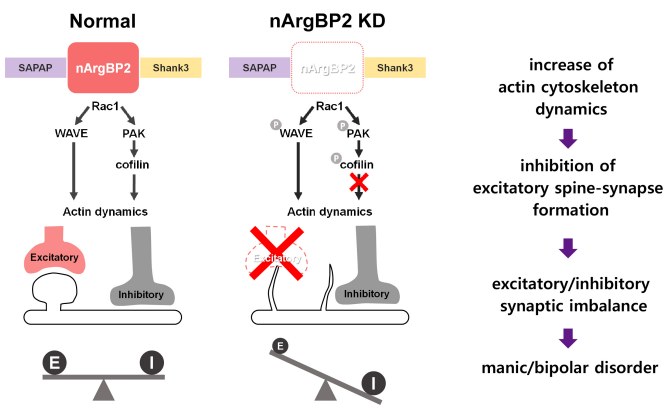

Fig. 1. Schematic description of the role of $n A \operatorname{rgBP} 2$ in excitatory spine-synapse formation, and maintenance of the E/I balance. In normal condition, nArgBP2, which interacts with SAPAP and Shank3, controls actin cytoskeleton dynamics through WAVE and $\mathrm{PAK} /$ cofilin cascade. In nArgBP2 KD neurons, the increased level of actin cytoskeleton dynamics results in aberrant spine morphology. Subsequent inhibition of excitatory spine-synapse formation leads to excitatory/inhibitory synaptic imbalance, which may account for manic/bipolar-like behaviors observed in nArgBP2 knockout mice and analogous human neurological disorders.

As stated above, nArgBP2 knockout mice showed manic/ bipolar-like behavior including, but not limited to, increased activity, compulsive/repetitive behavior, risk-taking behavior, hedonistic and anti-depressant-like behavior, resembling many aspects of $\mathrm{BD}$. There was a significant reduction in the average number of spines per dendrite in individuals with $B D$, as compared with controls. Therefore, reduction in the number of mushroom-shaped dendritic spines and subsequent inhibition of the spine-synapse formation that we observed are largely consistent with the phenotypes observed in individuals with $\mathrm{BD}$, thus providing the neurobiological basis that links the reduction in expression of $\mathrm{nArgBP} 2$ with a synaptic dysfunction associated with BD. Furthermore, changes in the proportion of excitatory synaptic contacts, formed either on dendritic spines or shafts, controls neuronal efficacy; any alteration of this equilibrium could lead to various psychiatric disorders. Therefore, the morphological changes in synapses of nArgBP2 $\mathrm{KD}$ neurons that alter excitatory synapse formation without affecting the inhibitory synapses, may account for manic/ bipolar-like behaviors observed in nArgBP2 knockout mice.

In conclusion, since nArgBP2 binds to the two most prominent postsynaptic proteins that are associated with various mood disorders, we propose that nArgBP2could function as a hub able to link together etiological factors of different disorders. This certainly requires further detailed studies.

\section{ACKNOWLEDGEMENTS}

This research was supported by Grant NRF-2015M3C7A10 28790 from the Brain Research Program through the National Research Foundation of Korea, and Grant 20100029395 from the Biomembrane Plasticity Research Center funded by the Ministry of Science, ICT \& Future Planning, Republic of Korea. 